\title{
GROWTH PROPERTIES OF FUNCTIONS IN HARDY FIELDS
}

\author{
MAXWELL ROSENLICHT
}

\begin{abstract}
This paper continues the author's earlier work on the notion of rank in a Hardy field. Further results are given on functions in Hardy fields of finite rank, including extensions of Hardy's results on the rates of growth of his logarithmicoexponential functions.
\end{abstract}

1. Comparability classes. Nonzero elements $\alpha, \beta$ of an ordered abelian group are called comparable if there are positive integers $m, n$ such that $m|\alpha|>|\beta|$ and $n|\beta|>|\alpha|$. Nonzero elements $f, g$ of a Hardy field $k$ are accordingly called comparable if both $\lim _{x \rightarrow \infty} f(x)$ and $\lim _{x \rightarrow \infty} g(x)$ are 0 or $\pm \infty$ and their rates of approach to 0 or $+\infty$ or $-\infty$ as $x \rightarrow+\infty$ are comparable, that is if the nonzero elements $\nu(f), \nu(g)$ of the value group $\nu\left(k^{*}\right)$ of $k$ are comparable, as in [5, §3]. (We assume some familiarity with [5], whose notation we employ.) More generally, germs $f, g$ of continuous real-valued functions on positive half-lines in $\mathbf{R}$ which are nowhere zero on some half-line and are such that $\lim _{x \rightarrow \infty} f(x)$ and $\lim _{x \rightarrow \infty} g(x)$ are either 0 , or $+\infty$, or $-\infty$, will be called comparable if, on some half-line, each of $|f|,|g|$ is bounded above and below by suitable integral powers of the other. Comparability is an equivalence relation among such germs. In particular, comparability is an equivalence relation among all nonzero elements $f$ of Hardy fields with the property that $\lim _{x \rightarrow \infty} f(x)$ is one of $0,+\infty$, or $-\infty$, that is among all nonzero elements $f$ such that $\nu(f) \neq 0$.

For $f$ a germ of a nowhere zero continuous real-valued function on a positive half-line that approaches $0,+\infty$, or $-\infty$ as $x \rightarrow+\infty$, denote the comparability class of $f$ by $\mathrm{Cl}(f)$. Noting that $f,-f, 1 / f$, and $-1 / f$ are comparable and that precisely one of these is infinitely increasing (that is, approaches $+\infty$ ), we define $\mathrm{Cl}(f)<\mathrm{Cl}(g)$ if each infinitely increasing element of $\mathrm{Cl}(f)$ is less than each infinitely increasing element of $\mathrm{Cl}(g)$, "less than" meaning "less than on some half-line". If $f, g$ are nonzero elements of a Hardy field $k$ such that $\nu(f), \nu(g) \neq 0$, and $\mathrm{Cl}(f) \neq \mathrm{Cl}(g)$, then either $\mathrm{Cl}(f)<\mathrm{Cl}(g)$ or $\mathrm{Cl}(g)<\mathrm{Cl}(f)$. The rank of a Hardy field is the number of its comparability classes.

Proposition 1. If $u, v$ are infinitely increasing elements of a Hardy field, then $\mathrm{Cl}(u) \geqslant \mathrm{Cl}(v)$ if and only if $\nu\left(u^{\prime} / u\right) \leqslant \nu\left(v^{\prime} / v\right)$, which holds if and only if $\nu(\log u) \leqslant$ $\nu(\log v)$.

Received by the editors September 20, 1985.

1980 Mathematics Subject Classification (1985 Revision). Primary 34E05, 41A60; Secondary 12H05, 13N05, $26 \mathrm{~A} 12$.

Research supported by National Science Foundation grant number DMS- 8303286 . 
The first part of this result is a restatement of [5, Propositions 3, 4]; we do not even have to assume $u, v$ infinitely increasing here, just that $u, v \neq 0$ and $\nu(u)$, $\nu(v) \neq 0$, for one of $\pm u, \pm 1 / u$ is infinitely increasing and gives the same $\nu\left(u^{\prime} / u\right)$, and similarly for $v$. The last part follows from [5, lemma to Proposition 6].

Corollary. Let $u, v$ be nonzero elements of a Hardy field such that $\nu(u), \nu(v)$, $\nu(u / v) \neq 0$ and $\mathrm{Cl}(u / v)<\mathrm{Cl}(u)$. Then $\mathrm{Cl}(u)=\mathrm{Cl}(v)$ and $u^{\prime} / v^{\prime} \sim u / v$.

For $(u / v)^{\prime} /(u / v)=u^{\prime} / u-v^{\prime} / v$ and $\nu\left((u / v)^{\prime} /(u / v)\right)>\nu\left(u^{\prime} / u\right)$, so $u^{\prime} / u \sim$ $v^{\prime} / v$. Note that we would have obtained the same results, with simpler proofs, under the similar but stronger hypotheses $u, v \neq 0, \nu(u)=\nu(v) \neq 0$.

Proposition 2. Let $u$ be a nonzero element of a Hardy field, with $\nu(u) \neq 0$. Then

(1) if $\mathrm{Cl}(u)>\mathrm{Cl}(x)$ then $\nu\left(u^{\prime} / u\right)<\nu(1 / x), u^{\prime}, u^{\prime \prime}, \ldots, \nu\left(u^{\prime}\right), \nu\left(u^{\prime \prime}\right), \ldots$ are nonzero, $u^{\prime} / u \sim u^{\prime \prime} / u^{\prime} \sim \cdots, \log |u| \sim \log \left|u^{\prime}\right| \sim \cdots$, and, except in the case $\mathrm{Cl}(u)=\mathrm{Cl}\left(e^{x}\right), \nu\left(u^{\prime} / u\right) \neq 0$ and $\mathrm{Cl}\left(u^{\prime} / u\right)<\mathrm{Cl}(u)$.

(2) If $\mathrm{Cl}(u)=\mathrm{Cl}(x)$ then $\nu\left(u^{\prime} / u\right)=\nu(1 / x)$ and $\nu\left(u^{\prime \prime} / u^{\prime}\right) \geqslant \nu\left(u^{\prime} / u\right)$, with $u^{\prime \prime} / u^{\prime}+u^{\prime} / u$.

(3) If $\mathrm{Cl}(u)<\mathrm{Cl}(x)$ then $\nu\left(u^{\prime} / u\right)>\nu(1 / x), u^{\prime \prime} / u^{\prime} \sim-1 / x$ and either $\nu(u / \log x)=0$ or $\mathrm{Cl}\left(u^{\prime} x\right)<\mathrm{Cl}(x)$.

We have $u^{\prime} \neq 0$, for otherwise $u \in \mathbf{R}^{*}$ and $\nu(u)=0$, contrary to assumption. By Proposition 1 we have

$$
\mathrm{Cl}(u)\left\{\begin{array} { l } 
{ > } \\
{ = \mathrm { Cl } ( x ) } \\
{ < }
\end{array} \quad \text { according as } \nu ( u ^ { \prime } / u ) \left\{\begin{array}{l}
< \\
=\nu(1 / x), \\
>
\end{array}\right.\right.
$$

which is true according as

$$
\nu\left(u / u^{\prime}\right)\left\{\begin{array}{l}
> \\
=\nu(x) \\
<
\end{array}\right.
$$

which cases imply respectively that

$$
\nu\left(\left(u / u^{\prime}\right)^{\prime}\right)\left\{\begin{array} { l } 
{ > } \\
{ = \nu ( 1 ) = 0 , } \\
{ < }
\end{array} \text { or } \quad \nu ( ( u ^ { \prime } ) ^ { 2 } - u u ^ { \prime \prime } ) \left\{\begin{array}{l}
> \\
=\nu\left(\left(u^{\prime}\right)^{2}\right) . \\
<
\end{array}\right.\right.
$$

Note that $u^{\prime \prime}, u^{\prime \prime \prime}, \ldots$ are all nonzero, unless $u \in \mathbf{R}[x]$, which occurs only in case (2). Note also that the case $\nu\left(u^{\prime}\right)=0$ occurs only if $u^{\prime} \sim c$, for some $c \in \mathbf{R}^{*}$, in which case $u \sim c x$, so we are again in case (2). Therefore we have, in case (1), $\nu\left(\left(u^{\prime}\right)^{2}-u u^{\prime \prime}\right)>\nu\left(\left(u^{\prime}\right)^{2}\right)$, so $\left(u^{\prime}\right)^{2} \sim u u^{\prime \prime}, u^{\prime} / u \sim u^{\prime \prime} / u^{\prime} \sim \cdots$, with $\nu\left(u^{\prime}\right)$, $\nu\left(u^{\prime \prime}\right), \ldots \neq 0$ and $\log |u| \sim \log \left|u^{\prime}\right| \sim \ldots$. Also

$$
\nu\left(\left(u^{\prime} / u\right)^{\prime} /\left(u^{\prime} / u\right)\right)=\nu\left(u^{\prime \prime} / u^{\prime}-u^{\prime} / u\right)>\nu\left(u^{\prime} / u\right)
$$

so that if $\nu\left(u^{\prime} / u\right) \neq 0$ we have $\mathrm{Cl}\left(u^{\prime} / u\right)<\mathrm{Cl}(u)$; if $\nu\left(u^{\prime} / u\right)=0$ we have $\mathrm{Cl}(u)=$ $\mathrm{Cl}\left(e^{x}\right)$. In case $(2)$, we have $\nu\left(\left(u^{\prime}\right)^{2}-u u^{\prime \prime}\right)=\nu\left(\left(u^{\prime}\right)^{2}\right)$ and the stated claims follow immediately. In case (3), from $\nu\left(x u^{\prime}\right)>\nu(u) \neq 0$ follows $\nu\left(x u^{\prime \prime}+u^{\prime}\right)>\nu\left(u^{\prime}\right)$, or $x u^{\prime \prime} \sim-u^{\prime}$, so $u^{\prime \prime} / u^{\prime} \sim-1 / x$. If $\nu\left(u^{\prime} x\right)=0$ then there is a $c \in \mathbf{R}^{*}$ such that $u^{\prime} x \sim c$, or $u^{\prime} \sim c / x$, so $u \sim c \log x$, or $\nu(u / \log x)=0$, so if $\nu(u / \log x) \neq 0$ then $\nu\left(u^{\prime} x\right) \neq 0$ and $\nu\left(\left(u^{\prime} x\right)^{\prime} / u^{\prime} x\right)=\nu\left(u^{\prime \prime} / u^{\prime}+1 / x\right)>\nu(1 / x)$, so $\mathrm{Cl}\left(u^{\prime} x\right)<\mathrm{Cl}(x)$. 
COROllary 1. Let $u$ be a nonzero element of a Hardy field, with $\nu(u) \neq 0$, $\nu\left(u^{\prime}\right) \neq 0$, and $\mathrm{Cl}\left(u^{\prime}\right)>\mathrm{Cl}(x)$. Then $u \sim\left(u^{\prime}\right)^{2} / u^{\prime \prime}$.

In either case (2) or (3) we have $\nu\left(u^{\prime \prime} / u^{\prime}\right) \geqslant \nu(1 / x)$, contrary to the assumption that $\mathrm{Cl}\left(u^{\prime}\right)>\mathrm{Cl}(x)$, so case (1) obtains. (Note: This corollary justifies such classical computations as $\int e^{x^{2}} d x \sim\left(e^{x^{2}}\right)^{2} /\left(e^{x^{2}}\right)^{\prime}=e^{x^{2}} / 2 x$.)

It is convenient to define $\lambda(u)=u^{\prime} / u$ for any nonzero element $u$ of a Hardy field. Then $\nu(\lambda(u))$ depends only on $\mathrm{Cl}(u)$ if $\nu(u) \neq 0$, so that $\mathrm{Cl}(\lambda(u))$ depends only on $\mathrm{Cl}(u)$ if $\nu(\lambda(u)) \neq 0$, that is if $\mathrm{Cl}(u) \neq \mathrm{Cl}\left(e^{x}\right)$.

If $u$ is infinitely increasing then $\lambda(u)>0$. If, in addition, $\mathrm{Cl}(u)>\mathrm{Cl}\left(e^{x}\right)$, then $\nu(\lambda(u))=\nu\left(u^{\prime} / u\right)<\nu\left(\left(e^{x}\right)^{\prime} / e^{x}\right)=0$, so that $\lambda(u)$ is also infinitely increasing. If $u>v$ are infinitely increasing elements of a Hardy field then $\log u>\log v$, so that $\log u-\log v$ is either infinitely increasing or bounded from above, and therefore $\nu(\log u) \leqslant \nu(\log v)$. Taking derivatives we get $\nu(\lambda(u)) \leqslant \nu(\lambda(v))$, so that $\mathrm{Cl}(\lambda(u))$ $\geqslant \mathrm{Cl}(\lambda(v))$. Removing the restrictions that $u, v$ be infinitely increasing, we get the following result.

Corollary 2. Let $u, v$ be nonzero elements of a Hardy field such that $\nu(u)$, $\nu(v) \neq 0$ and $\mathrm{Cl}(u) \geqslant \mathrm{Cl}(v)>\mathrm{Cl}\left(e^{x}\right)$. Then $\mathrm{Cl}(\lambda(u)) \geqslant \mathrm{Cl}(\lambda(v))$.

COROllaRY 3. If $u$ is a nonzero element of a Hardy field such that $\nu(u)$, $\nu(\lambda(u)) \neq 0$, then $\mathrm{Cl}(\lambda(u))<\mathrm{Cl}(u)$ if and only if $\mathrm{Cl}(u)>\mathrm{Cl}(x)$, and in this case $\mathrm{Cl}\left(u^{\prime}\right)=\mathrm{Cl}(u)$. If $u$ is an element of a Hardy field such that $u>e^{x^{N}}$ for all real $N$, then $\mathrm{Cl}(\lambda(u))=\mathrm{Cl}(\log u)$.

For $\mathrm{Cl}(\lambda(u))<\mathrm{Cl}(u)$ if and only if $\nu\left(\left(u^{\prime} / u\right)^{\prime} /\left(u^{\prime} / u\right)\right)>\nu\left(u^{\prime} / u\right)$, which holds if and only if $\nu\left(\left(u^{\prime}\right)^{2}-u u^{\prime \prime}\right)>\nu\left(\left(u^{\prime}\right)^{2}\right)$, or $\left(u^{\prime}\right)^{2} \sim u u^{\prime \prime}$, proving the first contention. Since $\mathrm{Cl}\left(u^{\prime} / u\right)<\mathrm{Cl}(u)$, it follows that $\mathrm{Cl}\left(u^{\prime}\right)=\mathrm{Cl}(u)$. If $u>e^{x^{N}}$ for all real $N$, then $\mathrm{Cl}(\log u)>\mathrm{Cl}(x)$ and we can infer that $\mathrm{Cl}\left((\log u)^{\prime}\right)=\mathrm{Cl}(\log u)$, which means just $\mathrm{Cl}(\lambda(u))=\mathrm{Cl}(\log u)$.

Parts of these last results have also been proved by D. Gokhman (unpublished) and M. Boshernitzan [1, \$12].

\section{Level.}

Proposition 3. Let $k$ be $a$ Hardy field and $\Psi(k)=\left\{\nu\left(u^{\prime} / u\right): u \in k^{*}, \nu(u) \neq 0\right\}$. If $\Psi(k)=\varnothing$, then $k \subset \mathbf{R}$. For any $g \in k^{*}$ such that $\nu(g) \neq 0$ we have $\nu\left(g^{\prime}\right) \neq$ l.u.b. $\Psi(k)$. If $f \in k^{*}, \nu(f) \neq 1$.u.b. $\Psi(k)$, then there exists $g \in k^{*}$ such that $\nu(g) \neq 0$ and $f \sim g^{\prime}$, and $\nu(g)>0$ if and only if $\nu(f)$ exceeds each element of $\Psi(k)$.

If $\Psi(k)=\varnothing$ then for any $f \in k^{*}$ we have $\nu(f)=0$. In the Hardy field $k(x)$, where $x$ is the germ of the identity function on $\mathbf{R}$, we have $\nu(f)>\nu(x) \neq 0$, so that $\nu\left(f^{\prime}\right)>\nu\left(x^{\prime}\right)=\nu(1)=0$. Therefore $f^{\prime}=0$ and $f \in \mathbf{R}$. The next statement is precisely [5, Proposition 2]. If now $f \in k^{*}$ and $\nu(f) \neq 1$.u.b. $\Psi(k)$, then [5, Theorem 1] and the argument immediately preceding [6, Lemma 1] show that there exists some $g \in k^{*}$ such that $\nu(g) \neq 0$ and $f \sim g^{\prime}$. If $\nu(g)>0$ then [5, Proposition 1] shows that $\nu\left(g^{\prime}\right)$ exceeds each element of $\Psi(k)$. If $\nu(g)<0$ then $\nu(f)=\nu\left(g^{\prime}\right)=$ $\nu\left(g^{\prime} / g\right)+\nu(g)<\nu\left(g^{\prime} / g\right) \in \Psi(k)$. 
If $u$ is an infinitely increasing element of a Hardy field $k$, then its repeated logarithms $l_{1}(u)(=l(u)=\log u), l_{2}(u)(=\log \log u), l_{3}(u), \ldots$ are also infinitely increasing and $k\left(x, l_{1}(u), l_{2}(u), \ldots\right)$ is also a Hardy field, so that we can compare its comparability classes.

THEOREM 1. Let $k$ be a Hardy field of finite rank $r$ and let $u \in k$ be infinitely increasing. Then there is a integer $s$ with $|s| \leqslant r$ such that for all integers $N>r$ we have $l_{N}(u) \sim l_{N-s}(x)$.

For the proof we use repeatedly the fact that if $f$ is an infinitely increasing element of a Hardy field $k$ which has smaller comparability classes than $\mathrm{Cl}(f)$, then there is a $g \in k$ such that $g \sim \log f$ [5, Proposition 6]. As a consequence, if $u$ is an infinitely increasing element of an extension Hardy field of $k$ and $u \sim v$, for some $v \in k$, then either $\mathrm{Cl}(v)$ is the smallest comparability class of $k$ or $\log u \sim v_{1}$, for some $v_{1} \in k$. Since $u, l_{1}(u), l_{2}(u), \ldots$ are all infinitely increasing and mutually incomparable, there is an integer $i$, with $0 \leqslant i<r$, such that $l_{i}(u) \sim$ (some element of the smallest comparability class of $k)$. Now, since $k$ has finite rank, $\Psi(k)$ is finite. Suppose for a moment that $\max \Psi(k) \neq 0$. Taking $f=1$ in Proposition 3, there is a $g \in k$ such that $g^{\prime} \sim 1$, or $g \sim x$, so there exists an integer $j$, with $0 \leqslant j<r-1$ such that $l_{j}(x) \sim$ (some element of the smallest comparability class of $k$ ). Thus $\mathrm{Cl}\left(l_{i}(u)\right)=\mathrm{Cl}\left(l_{j}(x)\right)$ for some $i, j \in\{0,1, \ldots, r-1\}$. If however $\max \Psi(k)=0$ then there exists $v \in k^{*}$ such that $\nu(v) \neq 0$ and $\nu\left(v^{\prime} / v\right)=0=$ $\nu\left(\left(e^{x}\right)^{\prime} / e^{x}\right)$, and $\mathrm{Cl}(v)=\mathrm{Cl}\left(e^{x}\right)$ is the smallest comparability class of $k$. In this case we have $\mathrm{Cl}\left(l_{i}(u)\right)=\mathrm{Cl}\left(l_{j}(x)\right)$ with $j=-1$. In either case we have, by Proposition 1 , $\nu\left(l_{i+1}(u)\right)=\nu\left(l_{j+1}(x)\right)$, so that $l_{i+1}(u) \sim l_{j+1}(x)$, for some positive $c \in \mathbf{R}$, hence $l_{i+2}(u) \sim l_{j+2}(x), l_{i+3}(u) \sim l_{j+3}(x), \ldots$, which completes the proof.

We say that an infinitely increasing element $u$ of a Hardy field has level $s$ if for some integer $N$ we have $l_{N}(u) \sim l_{N-s}(x)$. Then $l_{N+1}(u) \sim l_{N+1-s}(x), l_{N+2}(u) \sim$ $l_{N+2-s}(x), \ldots$ so that the integer $s$, if it exists, is unique and the set of integers $N$ such that $l_{N}(u) \sim l_{N-s}(x)$ is closed under the taking of successors. The verification of the following properties of level is straightforward.

Proposition 4. For infinitely increasing elements $u, u_{1}, u_{2}$ of Hardy fields, level has the following properties.

(1) $e_{n}(x)$ has level $n, l_{n}(x)$ has level $-n$.

(2) If the Hardy field $\mathbf{Q}\langle u\rangle$ has rank $r$, then u has level $0, \pm 1, \ldots$, or $\pm r$.

(3) If $u_{1}, u_{2}$ are of levels $s_{1}, s_{2}$ respectively and $u_{1} \leqslant u_{2}$, then $s_{1} \leqslant s_{2}$.

(4) If $u_{1}, u_{2}$ have levels and are comparable, then their levels are equal.

(5) If $u_{1}, u_{2}$ lie in a common Hardy field and have levels $s_{1}, s_{2}$ respectively, then $u_{1}+u_{2}$ and $u_{1} u_{2}$ have level $\max \left\{s_{1}, s_{2}\right\}$.

(6) If $u_{1}, u_{2}$ have levels $s_{1}, s_{2}$ respectively and the composite germ $u_{1} \circ u_{2}$ lies in $a$ Hardy field, then $u_{1} \circ u_{2}$ has level $s_{1}+s_{2}$.

(7) If $u$ has level $s$ and the integer $N$ is such that $l_{N}(u) \sim l_{N-s}(x)$, then for each real number $\varepsilon>0$ we have $e_{N}\left((1-\varepsilon) l_{N-s}(x)\right)<u<e_{N}\left((1+\varepsilon) l_{N-s}(x)\right)$.

Clearly any transexponential function, that is an element of a Hardy field that exceeds any repeated exponential $e_{n}(x)$, has no level. Examples of such functions 
are given in [2], which also shows the existence of an infinitely increasing element $g$ of a Hardy field such that $g(g(x))=e^{x}$. This $g$ has no level, for if it had level $s$ then by (6) above $e^{x}$ would have level $2 s$.

Consider now germs of continuous functions on positive half-lines in $\mathbf{R}$ that are obtained by starting with the identity germ $x$ and taking repeatedly multiplies by positive real numbers, or exponentials, or logarithms, that is germs which are functional composites $\left(c_{1} e_{i_{1}}\right) \circ\left(c_{2} e_{i_{2}}\right) \circ \cdots \circ\left(c_{n} e_{i_{n}}\right)$, with each $c_{i}$ a positive real number and its occurrence in this expression denoting multiplication by $c_{i}$ and each $i_{j}$ an integer (so that, for example, $\left(1 e_{2}\right) \circ\left(c_{2} e_{-2}\right)(x)=e_{2}\left(c_{2} l_{2}(x)\right)=\exp \left((\log x)^{c_{2}}\right)$ ). These germs are infinitely increasing elements of a Hardy field containing them all and they form a group under functional composition. The question arises of comparing two such germs. If $g, g_{1}, g_{2}$ are such germs, then $g_{1}=g_{2}$, or $g_{1} \sim g_{2}$, or $g_{1}>g_{2}$, or $\mathrm{Cl}\left(g_{1}\right) \geqslant \mathrm{Cl}\left(g_{2}\right)$, or level $g_{1} \geqslant$ level $g_{2}$ if and only if $g_{1} \circ g=g_{2} \circ g$, or $g_{1} \circ g \sim g_{2} \circ g, \ldots$ respectively. The germ $\left(c_{1} e_{i_{1}}\right) \circ\left(c_{2} e_{i_{2}}\right) \circ \ldots \circ\left(c_{n} e_{i_{n}}\right)$ has level $i_{1}+$ $i_{2}+\cdots+i_{n}$. This germ can also be written in such a way that no $i_{j}$ is zero, except possibly $i_{n}$, and no $c_{j}$ is 1 , except possibly $c_{1}$. But there is no question of uniqueness of expression, since $\left(c_{1} e_{-1}\right) \circ\left(c_{2} e_{1}\right) \circ\left(c_{3} e_{0}\right)(x)=c_{1} \log c_{2}+c_{1} c_{3} x$ and different triples $\left(c_{1}, c_{2}, c_{3}\right)$ can give the same pair $\left(c_{1} \log c_{2}, c_{1} c_{3}\right)$; similarly for $\left(c_{1} e_{1}\right) \circ\left(c_{2} e_{-1}\right) \circ\left(c_{3} e_{0}\right)(x)=c_{1} c_{3}^{c_{2}} x^{c_{2}}$. However there are partial results.

Proposition 5. Consider expressions of the form $\left(c_{1} e_{i_{1}}\right) \circ\left(c_{2} e_{i_{2}}\right) \circ \cdots \circ\left(c_{n} e_{i_{n}}\right)$, representing germs of functions on positive half-lines in $\mathbf{R}$, with each $c_{i}$ a positive real number and each $i_{j}$ a nonnegative integer, such that no $c_{i}$ is 1 except possibly for $c_{1}$ and no $i_{j}$ is 0 except possibly for $i_{n}$. Two such distinct expressions represent comparable germs if and only if they are identical except for their $c_{1}$ 's and of two such expressions whose germs are not comparable the larger germ is the one for which the germ of $\left(1 e_{i_{1}-1}\right) \circ\left(c_{2} e_{i_{2}}\right) \circ \cdots \circ\left(c_{n} e_{i_{n}}\right)$ is larger, if both $i_{1}$ 's are positive.

These results, which enable us to compare any two expressions of the given type, are proved by applying induction on the maximal level $i_{1}+\cdots+i_{n}$ of the two expressions to both parts of the proposition simultaneously. We may assume that the two expressions under consideration have equal levels. The result is trivial for level zero. For positive level, each $i_{1}$ is positive and Proposition 1 tells us that the expression with the larger comparability class is that with the smaller $\nu\left(\log \left(\left(c_{1} e_{i_{1}}\right) \circ \cdots \circ\left(c_{n} e_{i_{n}}\right)(x)\right)\right)$, that is the one with the larger $\log \left(\left(c_{1} e_{i_{1}}\right) \circ \ldots \circ\left(c_{n} e_{i_{n}}\right)(x)\right)$, and $\log \left(\left(c_{1} e_{i_{1}}\right) \circ \cdots \circ\left(c_{n} e_{i_{n}}\right)(x)\right)$ $\left(e_{i_{1}-1} \circ\left(c_{2} e_{i_{2}}\right) \circ \cdots \circ\left(c_{n} e_{i_{n}}\right)\right)(x)$, which enables the induction to go through.

Taking functional inverses enables us to compare any two expressions of the form $\left(c_{1} l_{i_{1}}\right) \circ \cdots \circ\left(c_{n} l_{i_{n}}\right)$ if each $i_{j}$ is nonnegative.

COROLlaRY. Let $r_{1}, s_{1}, r_{2}, s_{2}$ be nonnegative integers and $c_{1}, c_{2}$ positive real numbers distinct from 1 , with $\left(r_{1}, s_{1}, c_{1}\right) \neq\left(r_{2}, s_{2}, c_{2}\right)$. Then

$$
e_{r_{1}}\left(\left(l_{s_{1}}(x)\right)^{c_{1}}\right)>e_{r_{2}}\left(\left(l_{s_{2}}(x)\right)^{c_{2}}\right)
$$


if and only if $r_{1}-s_{1}>r_{2}-s_{2}$ or

$$
\begin{aligned}
& r_{1}-s_{1}=r_{2}-s_{2}, r_{1}>r_{2}, \text { and } c_{1}>1, \text { or } \\
& r_{1}-s_{1}=r_{2}-s_{2}, r_{1}<r_{2}, \text { and } c_{2}<1, \text { or } \\
& r_{1}=r_{2}, s_{1}=s_{2} \text { and } c_{1}>c_{2} .
\end{aligned}
$$

Furthermore these two functions are comparable if and only if $r_{1}=r_{2}=0$ and $s_{1}=s_{2}$.

This useful result, which is given less explicitly in [3, pp. 23-24], reduces after the replacement of $x$ by $e_{s_{1}+s_{2}+1}(x)$ to the question of comparing $e_{r_{1}}\left(\left(e_{s_{2}+1}(x)\right)^{c_{1}}\right)=$ $\left(e_{r_{1}+1} \circ\left(c_{1} e_{s_{2}}\right)\right)(x)$ with $e_{r_{2}}\left(\left(e_{s_{1}+1}(x)\right)^{c_{2}}\right)=\left(e_{r_{2}+1} \circ\left(c_{2} e_{s_{1}}\right)\right)(x)$, which reduces directly to the proposition.

Hardy points out [3, p. 24] that for any real numbers $\alpha_{1}, \alpha_{2}, \alpha_{3}, \ldots, \beta_{1}, \beta_{2}, \beta_{3}, \ldots$ such that $0<\alpha_{1}, \alpha_{2}, \ldots<1<\beta_{1}, \beta_{2}, \ldots$ we have

$$
\begin{gathered}
e_{2}\left(\left(l_{1}(x)\right)^{\alpha_{1}}\right)>e_{3}\left(\left(l_{2}(x)\right)^{\alpha_{2}}\right)>\cdots>e_{r+1}\left(\left(l_{r}(x)\right)^{\alpha_{r}}\right)>\cdots \\
\ldots>e_{r}\left(\left(l_{r}(x)\right)^{\beta_{r}}\right)>\cdots>e_{2}\left(\left(l_{2}(x)\right)^{\beta_{2}}\right)>e_{1}\left(\left(l_{1}(x)\right)^{\beta_{1}}\right),
\end{gathered}
$$

and $e_{r+1}\left(\left(l_{r}(x)\right)^{\alpha_{r}}\right)>e_{r}\left(\left(l_{r}(x)\right)^{\beta_{r}}\right.$ ) for all $r$ (all direct consequences of the corollary). He also shows [4, pp. 82-85] that there is an infinitely increasing function $\phi$ such that $\phi(\phi(x))=e^{x}$ satisfying

$$
e_{r+1}\left(\left(l_{r}(x)\right)^{\alpha_{r}}\right)>\phi(x)>e_{r}\left(\left(l_{r}(x)\right)^{\beta_{r}}\right)
$$

for all $r$, and states without proof that such a function cannot be an $L$-function. We can prove that there is no function $\phi$ in a Hardy field of finite rank such that

$$
e_{r+1}\left(\left(l_{r}(x)\right)^{\alpha_{r}}\right)>\phi(x)>e_{r}\left(\left(l_{r}(x)\right)^{\beta_{r}}\right)
$$

for all $r$ as follows. Let $N, s$ be integers such that $l_{N}(\phi) \sim l_{N-s}(x)$. Taking $r=N$ above and applying the operator $l_{N}$ to the resulting inequalities shows that $l_{N-s}(x) / e\left(\left(l_{N}(x)\right)^{\alpha_{N}}\right)$ and $\left(l_{N}(x)\right)^{\beta_{N}} / l_{N-s}(x)$ are bounded as $x \rightarrow+\infty$. The corresponding expressions with $\alpha_{N}, \beta_{N}$ replaced by 1 are therefore bounded, giving respectively the estimates $s \leqslant 1$ and $s \geqslant 0$, so that $s=0$ or 1 , and both these possibilities can be ruled out directly by the same boundedness argument, with $\alpha_{N}, \beta_{N}$ this time maintaining their original values.

\section{Liouvillian Hardy fields.}

Proposition 6. Let $k \subset K$ be Hardy fields and let $w \in K^{*}$ be such that $\nu(w) \geqslant 0$ and $\nu\left(w^{\prime}\right) \in \nu\left(k^{*}\right)$. Then there exists a $t \in k^{*}$ such that $\nu(t)>0$ and $\nu\left(w^{\prime}\right) \geqslant \nu\left(t^{\prime}\right)$ if and only if $\nu\left(w^{\prime}\right) \neq$ l.u.b. $\Psi(k)$. This last condition is satisfied if l.u.b. $\Psi(k)$ does not exist or if $\max \Psi(k)$ exists.

If such a $t$ exists and $\nu\left(w^{\prime}\right)=1$ u.b. $\Psi(k)$ then $\nu\left(w^{\prime}\right) \geqslant \nu\left(t^{\prime}\right)$, which exceeds each element of $\Psi(k)$ [5, Proposition 1], so that $\nu\left(t^{\prime}\right) \geqslant 1$.u.b. $\Psi(k)=\nu\left(w^{\prime}\right)$, so that $\nu\left(w^{\prime}\right)=\nu\left(t^{\prime}\right)=$ l.u.b. $\Psi(k)$, contrary to [5, Proposition 2]. Conversely, suppose $\nu\left(w^{\prime}\right) \neq$ l.u.b. $\Psi(k)$, if this exists. We have $\nu\left(w^{\prime}\right) \in \nu\left(k^{*}\right)$ and $\nu\left(w^{\prime}\right)$ exceeds each element of $\Psi(k)$, hence each element of $\Psi(k)$, so by Proposition 3 there is a $t \in k^{*}$ such that $\nu(t)>0$ and $\nu\left(w^{\prime}\right)=\nu\left(t^{\prime}\right)$. This shows the equivalence of the two 
conditions. The last condition is already satisfied if l.u.b. $\Psi(k)$ does not exist. If, on the other hand, $\max \Psi(k)$ exists, then [5, Proposition 1] implies that $\nu\left(w^{\prime}\right)$ exceeds each element of $\Psi(K)$, in particular the element $\max \Psi(k)=$ l.u.b. $\Psi(k)$.

Proposition 7. Let $k \subset k(w)$ be Hardy fields, with either $w^{\prime} \in k$ or $w^{\prime} / w \in k$ and $w \sim 1$. Suppose that there exists $t \in k^{*}$ such that $\nu(t)>0$ and $\nu\left(w^{\prime}\right) \geqslant \nu\left(t^{\prime}\right)$. Then for each $u \in(k(w))^{*}$ there exists $v \in k^{*}$ such that $u \sim v$.

This result strengthens [6, Lemma 2], which under exactly the same hypotheses proves the weaker result that $\nu\left((k(w))^{*}\right) \subset \mathbf{Q} \nu\left(k^{*}\right)$. From this follows that if $u \in(k(w))^{*}$ then there exists a positive integer $n$ and an element $v \in k^{*}$ such that $\nu\left(u^{n}\right)=n \nu(u)=\nu(v)$. If $\nu(u) \neq 0$, we get $\nu\left(u^{\prime} / u\right)=\nu\left(\left(u^{n}\right)^{\prime} / u^{n}\right)=\nu\left(v^{\prime} / v\right) \in$ $\Psi(k)$. Thus $\Psi(k(w))=\Psi(k)$. We now prove the preliminary result that $\nu\left((k(w))^{*}\right)$ $\subset \nu\left(k^{*}\right)$. In the case $w^{\prime} \in k$, it suffices to prove by induction on $n$ that if $a_{0}, a_{1}, \ldots, a_{n} \in k$ and $u=a_{0} w^{n}+a_{1} w^{n-1}+\cdots+a_{n} \neq 0$, then $\nu(u) \in \nu\left(k^{*}\right)$. This is clearly true if $n=0$, so that we may assume that $n>0$. Also assume, as we may, that $a_{0}=1$. Then $u^{\prime} \in k[w]$ has degree in $w$ at most $n-1$, so that by induction either $u^{\prime}=0$, in which case $u \in \mathbf{R}^{*}$ and $\nu(u)=0$, or $\nu\left(u^{\prime}\right) \in \nu\left(k^{*}\right)$, in which case, unless $\nu(u)=0$, we have $\nu(u)=\nu\left(u^{\prime}\right)-\nu\left(u^{\prime} / u\right)$. But since $\nu\left(u^{\prime}\right) \in \nu\left(k^{*}\right)$ and $\nu\left(u^{\prime} / u\right) \in \Psi(k) \subset \nu\left(k^{*}\right)$ we get the desired inclusion $\nu(u) \subset \nu\left(k^{*}\right)$. In the case $w^{\prime} / w \in k$ it suffices to prove by induction on $\operatorname{card}\{i\}$ that a sum $u=\sum_{i} a_{i} w^{i}$, with the $i$ 's distinct integers and each $a_{i} \in k^{*}$, is either zero or such that $\nu(u) \in \nu\left(k^{*}\right)$. We may clearly assume that $n>0$ and that one of the terms $a_{i} w^{i}$ of $u$ is one. Noting that $\left(a_{i} w^{i}\right)^{\prime}=\left(a_{i}^{\prime} / a_{i}+i w^{\prime} / w\right) a_{i} w^{i}$, we see that $u^{\prime}$ is a sum of terms of the given type but with fewer terms, so by induction we get either $u^{\prime}=0$, in which case $u \in \mathbf{R}$ and either $u=0$ or $\nu(u)=0$, or $\nu\left(u^{\prime}\right) \in \nu\left(k^{*}\right)$, and if $\nu(u) \neq 0$ we get as before $\nu(u)=\nu\left(u^{\prime}\right)-\nu\left(u^{\prime} / u\right) \in \nu\left(k^{*}\right)$. This in any case $\nu\left((k(w))^{*}\right)=\nu\left(k^{*}\right)$. In particular, l.u.b. $\Psi(k(w))=$ l.u.b. $\Psi(k)$. We now complete the proof by reworking, somewhat differently, the previous argument. If $w^{\prime} \in k$ we show by induction on $n$ that for any $u=a_{0} w^{n}+a_{1} w^{n-1}+\cdots+a_{n}$, with each $a_{i} \in k$, we have either $u=0$ or $u \sim v$, for some $v \in k^{*}$. As before, we may assume $n>0$ and $a_{0}=1$. By the induction assumption $u-w^{n}$ is either 0 , in which case $u \sim 1$, or $u-w^{n} \sim b$, for some $b \in k^{*}$. In the latter case $u \sim b+1$, unless $b \sim-1$. If $b \sim-1$, then $u=0$ or $u \neq 0$ and $\nu(u)>0$. Then $\nu\left(u^{\prime}\right) \neq$ l.u.b. $\Psi(k)$, so there exists an $f \in k^{*}$ such that $\nu(f)>0$ and $f^{\prime} \sim u^{\prime}$, in which case $f \sim u$. In the case $w^{\prime} / w \in k$, we show by induction on $\operatorname{card}\{i\}$ that for any sum $u=\sum_{i} a_{i} w^{i}$, with the $i$ 's distinct integers and each $a_{i} \in k^{*}$, we have $u=0$ or $u \sim v$ for some $v \in k^{*}$. We may clearly assume that $u$ has more than one term and that one of its terms is 1 . The induction hypothesis then gives $u-1=0$, in which case $u=1$, or $u-1 \sim b$, for some $b \in k^{*}$. Then $u \sim 1+b$, unless $b \sim-1$, in which case $u=0$ or $u \neq 0$ and $\nu(u)>0$. As before, $f^{\prime} \sim u^{\prime}$ for some $f \in k^{*}$ such that $\nu(f)>0$ and as before we obtain $f \sim u$.

Proposition 8. Let $\mathbf{R} \subset k \subset K$ be Hardy fields and let $U \subset K$ be such that each $u \in U$ is primitive over $k$, that is $u^{\prime} \in k$, and $K=k(U)$. If l.u.b. $\Psi(k)$ exists, suppose that there is a $u_{0} \in U$ such that $u_{0} \neq 0, \nu\left(u_{0}\right) \neq 0$, and $\nu\left(u_{0}^{\prime}\right)=1$. u.b. $\Psi(k)$. 
Then $\mathrm{Cl}\left(u_{0}\right)$ is smaller than any comparability class of $k$ and for each element $y$ of $K^{*}$ we have $y \sim \alpha u_{0}^{m}$ for some $\alpha \in k^{*}$ and $m \in \mathbf{Z}$. If 1.u.b. $\Psi(k)$ does not exist, then for each $y \in K^{*}$ there is an $\alpha \in k^{*}$ such that $y \sim \alpha$.

Suppose for a moment that l.u.b. $\Psi(k)$ exists. If $y \in k^{*}$ and $\nu(y)>0$ then $\nu\left(y^{\prime}\right)>$ l.u.b. $\Psi(k)=\nu\left(u_{0}^{\prime}\right)>\nu\left((1 / y)^{\prime}\right)$, so that $\nu(y)>\nu\left(u_{0}\right)>\nu(1 / y)$ and $\left|\nu\left(u_{0}\right)\right|<\nu(y)$. For any odd positive integer $N$ we can apply this same argument to the Hardy fields $k\left(y^{1 / N}\right) \subset k\left(U, y^{1 / N}\right)$ and the element $y^{1 / N}$ to obtain $\left|\nu\left(u_{0}\right)\right|<$ $\nu\left(y^{1 / N}\right)=(1 / N) \nu(y)$. Therefore $\mathrm{Cl}\left(u_{0}\right)<\mathrm{Cl}(y)$. Note that if there exists a $y \in k^{*}$ such that $\nu(y) \neq 0$ and $\nu\left(y^{\prime} / y\right)=$ l.u.b. $\Psi(k)$ then this $y$ could be chosen to be infinitely increasing and we would have an example of the present situation with $u_{0}=\log y$; in fact we must always have $u_{0} \sim c \log y$ for some $c \in \mathbf{R}^{*}$. Now in either case, l.u.b. $\Psi(k)$ existing or not, we consider the differential ring $k[U]$, whose field of quotients is $K$. Each nonzero element $y$ of $k[U]$ can be written as a finite sum of monomials $a u_{1} u_{2} \cdots u_{n}$, with $a \in k^{*}$ and $u_{1}, u_{2}, \ldots, u_{n} \in U$ and we call a positive integer $N$ the degree of $y$ if $y$ can be written as a sum of such monomials with each $n$ at most $N$, but not as such a sum with each $n$ at most $N-1$. We prove the proposition by proving by induction on the degree of the nonzero $y \in k[U]$ that if l.u.b. $\Psi(k)$ exists then $y \sim \alpha u_{0}^{m}$ for some $\alpha \in k^{*}$ and some nonnegative integer $m$, while if l.u.b. $\Psi(k)$ does not exist then $y \sim \alpha$, for some $\alpha \in k^{*}$; this will complete the proof since $K$ is the quotient field of $k[U]$. This statement is clearly true if $y$ has degree zero. So suppose that the degree of $y$ is $N>0$ and that the statement is true for each nonzero element of $k[U]$ of degree less than $N$. We shall prove our contention for all nonzero $y \in k[U]$ of degree $N$ by induction on the mininal number of monomials $a u_{1} \cdots u_{N}$ of degree $N$ that appear in any expression of $y$ as a sum of monomials of degree at most $N$. We have reached the point where we have positive integers $N, M$ and we want to show that for any nonzero $y \in k[U]$ that can be written as a sum of monomials of degree at most $N$ with at most $M$ monomials of degree $N$ appearing we have $y \sim \alpha u_{0}^{m}$ with $\alpha \in k^{*}$ and $m$ a nonnegative integer or $y \sim \alpha$ with $\alpha \in k^{*}$, as the case may be, under the assumption that this is true for any nonzero $y \in k[U]$ that can be written as a sum of monomials of degree at most $N$ with at most $M-1$ monomials of degree $N$ appearing. We may suppose that $y$ can be written as a sum of monomials of degree at most $N$ with exactly $M$ monomials of degree $N$ appearing and we may also assume, without loss of generality, that one of the terms of degree $M$ that actually appears in such a representation is of the form $u_{1} u_{2} \cdots u_{N}$, for some $u_{1}, \ldots, u_{N} \in U$. Then $y^{\prime}$ can be written as a sum of monomials of degree at most $N$ with less than $M$ monomials of degree $N$ appearing. If $y^{\prime}=0$ then $y \in \mathbf{R}^{*}$ and we are done. Otherwise, the induction assumption applied to $y^{\prime}$ shows that if l.u.b. $\Psi(k)$ exists then $y^{\prime} \sim \beta u_{0}^{p}$ for some $\beta \in k^{*}$ and some nonnegative integer $p$ while if l.u.b. $\Psi(k)$ does not exist then $y^{\prime} \sim \beta$ for some $\beta \in k^{*}$. We may assume that $\nu(y) \neq 0$, for otherwise $y \sim c$ for some $c \in \mathbf{R}^{*} \subset k$. If 1.u.b. $\Psi(k)$ does not exist then Proposition 3 implies that $\beta \sim \alpha^{\prime}$ for some $\alpha \in k^{*}$ such that $\nu(\alpha) \neq 0$; thus $y^{\prime} \sim \alpha^{\prime}$ so that $y \sim \alpha$, as claimed. For the rest of the proof we may restrict ourselves to the case in which l.u.b. $\Psi(k)$ 
exists, where $y^{\prime} \sim \beta u_{0}^{p}$. If $\nu(\beta) \neq$ l.u.b. $\Psi(k)$ then there is an $\alpha \in k^{*}$ such that $\nu(\alpha) \neq 0$ and $\beta \sim \alpha^{\prime}$. We already know that $\mathrm{Cl}\left(u_{0}\right)<\mathrm{Cl}(\alpha)$, so that $\nu\left(\alpha u_{0}^{p}\right) \neq 0$, $\nu\left(u_{0}^{\prime} / u_{0}\right)>\nu\left(\alpha^{\prime} / \alpha\right)$, and $\left(\alpha u_{0}^{p}\right)^{\prime}=\left(\alpha^{\prime} / \alpha+p u_{0}^{\prime} / u_{0}\right) \alpha u_{0}^{p} \sim \alpha^{\prime} u_{0}^{p} \sim \beta u_{0}^{p} \sim y^{\prime}$, giving $y \sim \alpha u_{0}^{p}$. In the final case $\nu(\beta)=1$.u.b. $\Psi(k)=\nu\left(u_{0}^{\prime}\right)$ we have $\beta \sim c u_{0}^{\prime}$ for some $c \in \mathbf{R}^{*}$. Taking $\alpha=c /(p+1)$, we get $\nu\left(\alpha u_{0}^{p+1}\right) \neq 0$ and

$$
\left(\alpha u_{0}^{p+1}\right)^{\prime}=\alpha(p+1) u_{0}^{p} u_{0}^{\prime} \sim \beta u_{0}^{p} \sim y^{\prime},
$$

so that $y \sim \alpha u_{0}^{p+1}$, completing the proof.

Proposition 9. Let $\mathbf{R} \subset k \subset K$ be Hardy fields and let $W$ be a multiplicative subgroup of $K^{*}$ that contains $k^{*}$ and is such that $K=k(W)$ and each $w \in W$ is exponential over $k$, that is $w^{\prime} / w \in k$. Suppose also that either $\max \Psi(k)$ exists or 1.u.b. $\Psi(k)$ does not exist. Then for each $y \in K^{*}$ there is $a w \in W$ such that $y \sim w$.

The set of all finite sums $w_{1}+\cdots+w_{n}$, where $w_{1}, \ldots, w_{n} \in W$, consists of the differential ring $k[W]$ whose field of quotients is $K$. It therefore suffices to prove the proposition for nonzero elements $y=w_{1}+\cdots+w_{n}$, where each $w_{i} \in W$, and we do this by induction on $n$. The case $n=1$ is trivial, so suppose that $n>1$ and that the result is true for $n-1$. Then $y=w_{1}+\cdots+w_{n} \sim w_{i}$ if $i \in\{1, \ldots, n\}$ is such that $\nu\left(w_{j}\right)>\nu\left(w_{i}\right)$ for all $j \in\{1, \ldots, n\}, j \neq i$. We are therefore reduced to the case where $\nu\left(w_{1}\right), \ldots, \nu\left(w_{n}\right)$ are not all distinct, say $\nu\left(w_{1}\right)=\nu\left(w_{2}\right)$. Let $c \in \mathbf{R}^{*}$ be such that $w_{2} \sim c w_{1}$, so that $w_{0}=w_{2} / c w_{1} \sim 1$. Applying Proposition 7 to $w_{0}$, taking note of Proposition 6, we see that for each nonzero $u \in k\left(w_{0}\right)$ there is a $v \in k^{*}$ such that $u \sim v$. We have $\nu\left(\left(k\left(w_{0}\right)\right)^{*}\right)=\nu\left(k^{*}\right), \Psi\left(k\left(w_{0}\right)\right)=\Psi(k), \max \Psi\left(k\left(w_{0}\right)\right)$ $=\max \Psi(k)$, and l.u.b. $\Psi\left(k\left(w_{0}\right)\right)=$ l.u.b. $\Psi(k)$, if these latter exist. If we apply our induction assumption to $y=w_{1}+\cdots+w_{n}=w_{1}\left(1+c w_{0}\right)+w_{3}+\cdots+w_{n}$, with $k$ replaced by $k\left(w_{0}\right), K$ by $K\left(w_{0}\right)$ and $W$ by $\left(k\left(w_{0}\right)\right)^{*} W$ we obtain $y \sim u w$, with $u \in\left(k\left(w_{0}\right)\right)^{*}$ and $w \in W$. If $u \sim v$, with $v \in k^{*}$, we have $y \sim v w \in W$.

Proposition 10. Let $k$ be a Hardy field containing $\mathbf{R}$ and let $W=\left\{ \pm \exp \left(\int a\right): a\right.$ $\in k\}$ be the set of all germs that are exponential over $k$, where $\int$ denotes antidifferentiation, so that $k(W)$ is a Hardy field. Let $w=\exp \left(\int a\right)$, where $a \in k$. If $a=0$ or $\nu(a)$ exceeds each element of $\Psi(k)$, then $\nu(w)=0$. If $\nu(a)$ is less than some element of $\Psi(k)$, then $a \sim b^{\prime}$ for some $b \in k^{*}$ such that $\nu(b)<0$ and either $\mathrm{Cl}\left(w / e^{b}\right)<$ $\mathrm{Cl}(w)$ or $\nu\left(w / e^{b}\right)=0$, with $\mathrm{Cl}(w)=\mathrm{Cl}\left(e^{b}\right)$ exceeding at least one comparability class of $k$. If $k$ has a smallest comparability class and $w_{0} \in k$ is one of its infinitely increasing elements and $a \in k^{*}$ is such that $a \sim c w_{0}^{\prime} / w_{0}$ for some $c \in \mathbf{R}^{*}$, then $\nu(w)=\nu\left(w_{0}^{c}\right)$; furthermore, in this last case $\mathrm{Cl}\left(w_{0}\right)$ is the smallest comparability class of $k(W)$.

We note first that $a=w^{\prime} / w$ is equivalent to $a=(\log |w|)^{\prime}$, or $w= \pm \exp \left(\int a\right)$. Since antiderivatives and exponentials of elements of a Hardy field lie in a larger Hardy field, $k(W)$ is itself a Hardy field. Now let $a \in k$ and $w=\exp \left(\int a\right)$. If $a=0$ then $w^{\prime}=0$, so $w \in \mathbf{R}^{*}$ and $\nu(w)=0$. If $a \neq 0$ and $\nu(a)$ exceeds each element of $\Psi(k)$ then we cannot have $\nu(w) \neq 0$, for otherwise $\nu(a)=\nu\left(w^{\prime} / w\right) \in$ $\Psi(k)$. We now claim that in each of the remaining cases we cannot have $\nu(w)=0$. 
For otherwise, choosing $\alpha \in \mathbf{R}^{*}$ such that $\nu(w-\alpha)>0$, we have $\nu(a)=\nu\left(w^{\prime} / w\right)$ $=\nu\left(w^{\prime}\right)=\nu\left((w-\alpha)^{\prime}\right)$, which by Proposition 3 exceeds each element of $\Psi(k(W))$ $\supset \Psi(k)$. If $\nu(a)$ is less than some element of $\Psi(k)$, then Proposition 3 shows the existence of some $b \in k^{*}$ such that $a \sim b^{\prime}$ and $\nu(b)<0$. Then $\nu\left(e^{b}\right) \neq 0$ and $\left(w / e^{b}\right)^{\prime} /\left(w / e^{b}\right)=w^{\prime} / w-b^{\prime}=a-b^{\prime}$, so that $\nu\left(\left(w / e^{b}\right)^{\prime} /\left(w / e^{b}\right)\right)=\nu\left(a-b^{\prime}\right)>$ $\nu(a)=\nu\left(w^{\prime} / w\right)$. This shows that $\mathrm{Cl}\left(w / e^{b}\right)<\mathrm{Cl}(w)$ or that $\nu\left(w / e^{b}\right)=0$, and also that $\mathrm{Cl}(w)$ exceeds at least one comparability class of $k$. In the final case, in which $a \sim c w_{0}^{\prime} / w_{0}$, with $a \in k^{*}$ and $w_{0}$ and $c$ as stated, we have

$$
\nu\left(\left(w / w_{0}^{c}\right)^{\prime} /\left(w / w_{0}^{c}\right)\right)=\nu\left(w^{\prime} / w-c w_{0}^{\prime} / w_{0}\right)>\nu\left(w_{0}^{\prime} / w_{0}\right)=\max \Psi(k),
$$

so that the first part of the proposition implies $\nu\left(w / w_{0}^{c}\right)=0$. Among other things, we have shown that $\mathrm{Cl}\left(w_{0}\right)$ is the smallest comparability class among $\{\mathrm{Cl}(w): w \in W$, $\nu(w) \neq 0\}$. Proposition 9 states that for each $y \in(k(W))^{*}$ there is a $w \in W$ such that $y \sim w$, which shows that $\mathrm{Cl}\left(w_{0}\right)$ is the smallest comparability class of $k(W)$.

THeOREM 2. Let $\mathbf{R} \subset k \subset K$ be Hardy fields, let $U \subset K$ consist of elements that are primitive over $k$, let $W$ be a multiplicative subgroup of $K^{*}$ that contains $k$ and that consists of elements that are exponential over $k$, and suppose that $K=k(U, W)$. Suppose also that either $\max \Psi(k)$ exists and there is a $u_{0} \in U$ such that $u_{0} \neq 0$, $\nu\left(u_{0}\right) \neq 0$ and $\nu\left(u_{0}^{\prime}\right)=\max \Psi(k)$, or that l.u.b. $\Psi(k)$ does not exist. If $\max \Psi(k)$ exists then for each $y \in K^{*}$ there are $m \in \mathbf{Z}$ and $w \in W$ such that $y \sim u_{0}^{m} w$. If l.u.b. $\Psi(k)$ does not exist, then for each $y \in K^{*}$ there is a $w \in W$ such that $y \sim w$.

If $\max \Psi(k)$ exists, Proposition 8 shows that $\mathrm{Cl}\left(u_{0}\right)$ is the smallest comparability class of $k(U)$ and that $\max \Psi(k(U))>\max \Psi(k)$. We now apply Proposition 9 to the Hardy fields $\mathbf{R} \subset k(U) \subset K$ and the multiplicative group $W_{1}=(k(U))^{*} W$, getting $\mathrm{Cl}\left(u_{0}\right)$ to be the smallest comparability class of $K$ and obtaining for each $y \in K^{*}$ a $y_{1} \in(k(U))^{*}$ and a $w_{1} \in W$ such that $y \sim y_{1} w_{1}$. By Proposition 8 again, $y_{1} \sim \alpha u_{0}^{m}$ for some $\alpha \in k^{*}$ and $m \in \mathbf{Z}$, so that $y \sim u_{0}^{m}\left(\alpha w_{1}\right)$. This finishes the case where $\max \Psi(k)$ exists. The same proof, simplified by omitting all references to $\mathrm{Cl}\left(u_{0}\right)$, handles the remaining case in which l.u.b. $\Psi(k)$ does not exist.

COROllary. Let $\mathbf{R} \subset k \subset K$ be Hardy fields, let $U \subset K$ consist of elements that are primitive over $k$, let $W$ be a multiplicative subgroup of $K^{*}$ that contains $k$ and that consists of elements that are exponential over $k$, and suppose that $K=k(U, W)$. Suppose that $k$ has a smallest comparability class and that $w_{0} \in k$ is one of its infinitely increasing elements. If $y \in K^{*}$ then either there exists some $b \in k$ such that $|b|$ is infinitely increasing, $\mathrm{Cl}\left(e^{b}\right)>\mathrm{Cl}\left(w_{0}\right)$, and either $\mathrm{Cl}\left(y / e^{b}\right)<\mathrm{Cl}(y)$ or $\nu\left(y / e^{b}\right)=0$, or else there exist $\alpha \in \mathbf{R}^{*}, m \in \mathbf{Z}$ and $c \in \mathbf{R}$ such that $y \sim \alpha\left(\log w_{0}\right)^{m} w_{0}^{c}$.

Since $\mathrm{Cl}\left(w_{0}\right)$ is the smallest comparability class of $k$ we have $\max \Psi(k)$ $=\nu\left(w_{0}^{\prime} / w_{0}\right)$. The infinitely increasing germ $\log w_{0}$ is primitive over $k$ and $\nu\left(\left(\log w_{0}\right)^{\prime}\right)=\max \Psi(k)$, so that we can replace $U$ by $U \cup\left\{\log w_{0}\right\}$ and $K$ by 
$k\left(U \cup\left\{\log w_{0}\right\}, W\right)$ if necessary to guarantee that we are in the circumstances of Theorem 2, with $u_{0}=\log w_{0}$. If $y \in K^{*}$ then $y \sim\left(\log w_{0}\right)^{m} w$ for some $m \in \mathbf{Z}$ and $w \in W$. The various possibilities for $w$ are enumerated in Proposition 10. In the first case, $\nu(w)=0$ so that $w \sim \alpha$ for some $\alpha \in \mathbf{R}^{*}$ and hence $y \sim \alpha\left(\log w_{0}\right)^{m}$. In the second case, there is a $b \in k^{*}$ such that $\nu(b)<0, \mathrm{Cl}\left(e^{b}\right)>\mathrm{Cl}\left(w_{0}\right)$, and either $\mathrm{Cl}\left(w / e^{b}\right)<\mathrm{Cl}(w)$ or $\nu\left(w / e^{b}\right)=0$, so that either $\mathrm{Cl}\left(y / e^{b}\right)<\mathrm{Cl}(w)=\mathrm{Cl}(y)$ or $\nu\left(y / e^{b}\right)=0$. In the final case, $\nu(w)=\nu\left(w_{0}^{c}\right)$ for some $c \in \mathbf{R}^{*}$, so there exists $\alpha \in \mathbf{R}^{*}$ such that $w \sim \alpha w_{0}^{c}$, giving $y \sim \alpha\left(\log w_{0}\right)^{m} w_{0}^{c}$.

In analogy with the work of Hardy on his logarithmico-exponential functions ( $L$-functions, for short), we pose the following definition: Let $k_{0}$ be the real algebraic closure of the Hardy field $\mathbf{R}(x)$ and for each $n>0$ let the Hardy field $k_{n}$ be the real algebraic closure of the Hardy field obtained by adjoining to $k_{n-1}$ all germs that are either primitive or exponential over $k_{n-1}$. ("Germ" of course means germ of real-valued functions on positive half-lines.)

A liouvillian Hardy field is a differential subfield of $\bigcup_{n=0}^{\infty} k_{n}$ that contains $\mathbf{R}$. Elements of $k_{n}$ that are not in $k_{n-1}$ are called liouvillian Hardy functions of order $n$. (Liouvillian Hardy functions generalize Hardy's $L$-functions and are somewhat easier to work with. In Hardy's definitions germs primitive over a field are replaced by germs that are logarithms of the absolute value of an element of the field and germs exponential over a field are replaced by germs that are the exponential of an element of the field.)

THEOREM 3. Let $u$ be an infinitely increasing liouvillian Hardy function of order $n$. If $n=0$ then $u \sim \alpha x^{m}$, for some positive real $\alpha$ and positive rational $m$. If $n>0$ then either $l_{n}(u) \sim \alpha x^{m}$, where $\alpha$ and $m$ are as above, or for some $i=1,2, \ldots, n$ we have $l_{n-i}(u) \sim \alpha\left(l_{i}(x)\right)^{m}\left(l_{i-1}(x)\right)^{c}$, where $\alpha, c \in \mathbf{R}, m \in \mathbf{Q}, \alpha>0$, and either $c>0$ or $c=0, m>0$.

The proof uses repeatedly the result [5, p. 665] that if $k \subset K$ are Hardy fields with $K$ algebraic over $k$, then $\nu\left(K^{*}\right) \subset \mathbf{Q} \nu\left(k^{*}\right)$. Since each infinitely increasing $u \in \mathbf{R}(x)$ satisfies $u \sim \alpha x^{m}$ for some positive real $\alpha$ and positive integer $m$, the case $n=0$ is clear. The proof for $n>1$ goes by induction on $n$, applying the last corollary to $k_{n-1}$ and its extension field obtained by adjoining all primitive and exponential germs over it and then using the quoted result on algebraic extensions.

A large number of minor results of Hardy [4] follow immediately. For example, the elements of the smallest comparability class of $k_{n}$ are precisely those $u$ $\alpha\left(l_{n}(x)\right)^{m}$, with $\alpha \in \mathbf{R}^{*}$ and $m \in \mathbf{Q}^{*}$. No liouvillian Hardy function of order 3 exists which exceeds all $e\left((l(x))^{n}\right), n=1,2, \ldots$, and is less than all $e_{2}\left((l(x))^{1 / n}\right)$. The function $x^{x^{x}}$ is of order 3. The function $x^{\pi}$ is a liouvillian Hardy function of order 1 but an $L$-function of order 2 .

\section{REFERENCES}

1. M. Boshernitzan, New “orders of infinity”, J. Analyse Math. 41 (1982), 130-167.

2. __ Hardy fields, existence of transexponential functions, and the hypertranscendence of solution to $g(g(x))=e^{x}$, Aequationes Math. $30(1986), 258-280$. 
3. G. H. Hardy, Orders of infinity, 2nd ed., Cambridge Univ. Press, London and New York, 1924.

4. , Properties of logarithmico-exponential functions, Proc. London Math. Soc. (2) 10 (1912), 54-90.

5. M. Rosenlicht, The rank of a Hardy field, Trans. Amer. Math. Soc. 280 (1983), 659-671.

6. Rank change on adjoining real powers to Hardy fields, Trans. Amer. Math. Soc. 284 (1984), 829-836.

Department of Mathematics, University of California, Berkeley, California 94720 